\title{
Factors influencing flow steadiness in laminar boundary layer shock interactions
}

Ozgur Tumuklu', Deborah A. Levin, Sergey F. Gimelshein, and Joanna M. Austin

Citation: 1786, 050005 (2016); doi: 10.1063/1.4967555

View online: http://dx.doi.org/10.1063/1.4967555

View Table of Contents: http://aip.scitation.org/toc/apc/1786/1

Published by the American Institute of Physics 


\title{
Factors Influencing Flow Steadiness in Laminar Boundary Layer Shock Interactions
}

\author{
Ozgur Tumuklu ${ }^{1, a)}$, Deborah A. Levin ${ }^{1}$, Sergey F. Gimelshein ${ }^{2}$ and Joanna M. \\ Austin ${ }^{3}$ \\ ${ }^{1}$ Department of Aerospace Engineering, University of Illinois at Urbana-Champaign \\ ${ }^{2}$ Department of Astronautical Engineering ,University of Southern California, Los Angeles \\ ${ }^{3}$ Department of Aerospace Engineering, California Institute of Technology \\ a)Corresponding author: tumuklu2@illinois.edu
}

\begin{abstract}
The Direct Simulation Monte Carlo method has been used to model laminar shock wave boundary interactions of hypersonic flow over a 30/55-deg double-wedge and "tick-shaped" model configurations studied in the Hypervelocity Expansion Tube facility and T-ADFA free-piston shock tunnel, respectively. The impact of thermochemical effects on these interactions by changing the chemical composition from nitrogen to air as well as argon for a stagnation enthalpy of $8.0 \mathrm{MJ} / \mathrm{kg}$ flow are investigated using the 2-D wedge model. The simulations are found to reproduce many of the classic features related to Edney Type V strong shock interactions that include the attached, oblique shock formed over the first wedge, the detached bow shock from the second wedge, the separation zone, and the separation and reattachment shocks that cause complex features such as the triple point for both cases. However, results of a reacting air flow case indicate that the size of the separation length, and the movement of the triple point toward to the leading edge is much less than the nitrogen case.
\end{abstract}

Keywords: Separated flow, hypersonic flow, DSMC, reacting air, velocity slip.

\section{INTRODUCTION}

In shock wave boundary layer interactions (SWBLI), transition from laminar to turbulence flow, and three-dimensional effects can be observed [1,2]. For design purposes, all these effects should be taken into consideration because of their significant impact on aerothermodynamic quantities such as heat transfer, skin friction and surface pressure coefficient. Indeed, these interactions may cause separated flows in the vicinity of the interaction region which could reduce the effectiveness of control surfaces and modify the heat transfer rate and pressure load substantially. The effects of nonequilibrium chemically reacting flow on SWBLI are studied extensively by Ballaro et al. [3] and Grumet et al. [4]. In particular, chemical reactions are found to increase the peak heat transfer rate especially in for the case of fully catalytic wall.

The Direct Simulation Monte Carlo (DSMC) method has also been used to model SWBLIs, but, to a lesser extent than CFD due to its higher computational expense. Using DSMC, Moss et al. [5] demonstrated the sensitivity of surface heat flux, skin friction, and size of the separation region to key DSMC numerical parameters and used the slip velocity as a numerical convergence criterion for double cone simulations. Markelov et al. [6] performed statistical simulations of the flow around the hollow cylinder flare and studied the effects of vibrational non-equilibrium in the free stream on the flow properties. By comparison of numerical results with the experiment [7], they showed that the DSMC method is capable of predicting separated flow properties in the near-continuum regime rather accurately if all of the numerical parameter requirements, such as time step, cell size, and the total number of particles, are satisfied. Moss and Bird [8] compared DSMC simulations for flow over a double cone configuration and obtained excellent agreement with heat flux measurements for a Knudsen number case about a factor of three larger than what will be presented in this work. Finally, more recent modeling and experiments [9] show that the impact of the slip velocity has a significant impact on the size of the separation region by comparing Navier-Stokes (NS) and DSMC solutions for a "tick" configuration in the near-continuum regime. The separation size predicted by NS is larger than DSMC due to the zero slip assumption which is an obvious shortcoming of the solution.

30th International Symposium on Rarefied Gas Dynamics AIP Conf. Proc. 1786, 050005-1-050005-8; doi: 10.1063/1.4967555

Published by AIP Publishing. 978-0-7354-1448-8/\$30.00 
TABLE 1. Free Stream Conditions

\begin{tabular}{lcc}
\hline \multicolumn{1}{c}{ Freestream Parameters: } & Double Wedge & "Tick" Model \\
\hline Mach number & 7.14 & 10.18 \\
Translational temperature, K & 710 & 156 \\
Static pressure, kPa & 0.78 & 0.29 \\
Velocity, m/s & 3812 & 2514 \\
Density, $\mathrm{kg} / \mathrm{m}^{3}$ & 0.0037 & 0.0064 \\
Number density, molec $/ \mathrm{m}^{3}$ & $7.96 \times 10^{22}$ & $1.35 \times 10^{23}$ \\
Stagnation enthalpy, MJ/kg & 8.0 & 3.1 \\
Unit Reynolds number, Re/m & $4.15 \times 10^{5}$ & $1.38 \times 10^{6}$ \\
Mean free path, m & $2.05 \times 10^{-5}$ & $9.587 \times 10^{-6}$ \\
Reference length, $\mathrm{m}$ & 0.0508 & 0.063 \\
Knudsen number & $4.08 \times 10^{-4}$ & $1.52 \times 10^{-4}$ \\
\hline
\end{tabular}

Two separation dominated flows have been studied by means of the DSMC approach in order to investigate the flow characteristics that have impact on the temporal steadiness, namely the required time for steady state and the factors that have effect on the flow field and surface parameters. In the first case, a shock dominated laminar flow over a double wedge is considered. Experiments were conducted in the Hypervelocity Expansion Tube (HET) by Swantek and Austin [10] and the impact of thermochemical effects on shock wave-boundary layer interaction by changing the chemical composition from nitrogen to air and the stagnation enthalpy of flow were investigated. The high-enthalpy nitrogen and reacting air cases shown in Figs. 1(a) and (b) are selected in this study. Secondly, the "tick" configuration are selected to model leading-edge separation [11]. In fact, this case is relatively easy to analyze due to the lack of the complicated boundary-layer development, as shown in Fig. 1(c). The experiments are being performed in the T-ADFA free-piston shock tunnel at UNSW Canberra [9, 12]. The current focus is to understand the origins of these laminar boundary layer shock interactions in terms of thermochemical non-equilibrium models, gas-surface interactions and separation by the comparisons of the experiments.

\section{FLOW CONDITIONS AND NUMERICAL PARAMETERS}

The Statistical Modeling In Low-density Environment (SMILE) [13] computational solver is used. SMILE employs the majorant frequency scheme for modeling the molecular collision frequency [14], the variable hard sphere (VHS) model [15] for modeling the interaction between particles and the total collision energy (TCE) approach [15] for modeling of the chemical reactions. The gas-surface interactions were modeled using the Maxwell model with full momentum and energy accommodation. The simulations presented in this paper assume 2-D geometries.

As shown in Table 1, the conditions for the current cases are in the near-continuum regime and the flow over the geometries involves multiple length scales and large density gradient. In fact, the density increases about 20 times in the vicinity of the transmission shock for the double wedge geometry, and about 12 times at the triple point for the "tick" model with respect to the freestream, as described in Fig 1. To satisfy the DSMC numerical parameter requirements and resolve large magnitude of gradient in the simulation domain, special attention was paid to the convergence of the DSMC numerical parameters so as not to impact the results. Figure 2(a) shows a comparison of the local mean free path to collision cell size ratio for the air case (see Table 2 for the details). As shown, the cell size is sufficiently small such that the above ratio is larger than unity throughout the domain. Similarly, for the nitrogen and argon cases of the wedge as well as the "tick" case, it was ensured that this ratio is larger than the unity. Since the number of collision cells required to obtain a resolution on the order of a mean free path is high, the corresponding number of simulated particles becomes prohibitively large for the cases. The air simulation was performed using more than 5.7 billion simulated particles and as shown in Fig. 2(b), the number of particles per collision cell in the shock region was found to be around 17 which is sufficient to model the chemical reactions. Similarly, the "tick" configuration was simulated using more than 1.1 billion simulated particles, the number of particles per collision cell throughout the domain was found to be around 18. For the non-reacting single species cases (i.e. argon and nitrogen), the number of particles per collision cell in the shock region was found to be around four. This number is sufficient since the majorant collision frequency algorithm is used in the selection of collision pairs in this work [14]. 


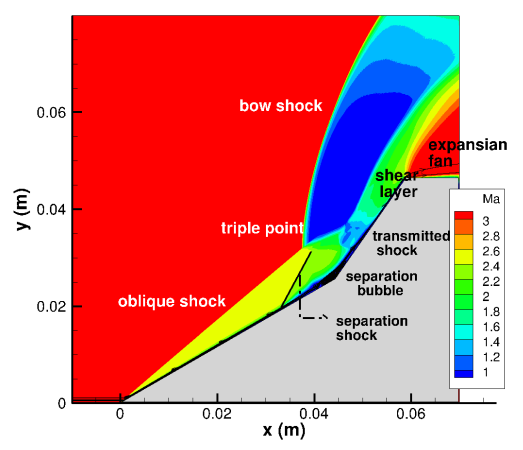

(a) Nitrogen case at $100 \mu \mathrm{s}$

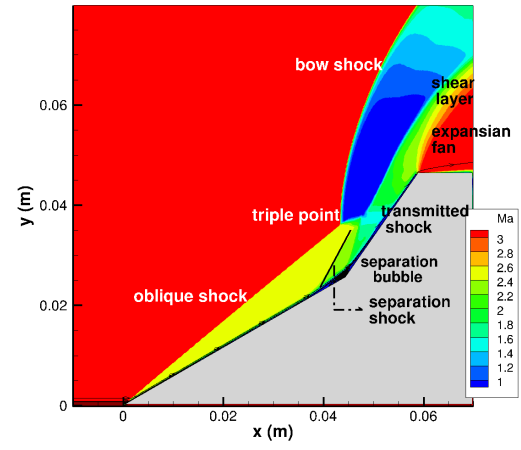

(b) Air case at $100 \mu \mathrm{s}$

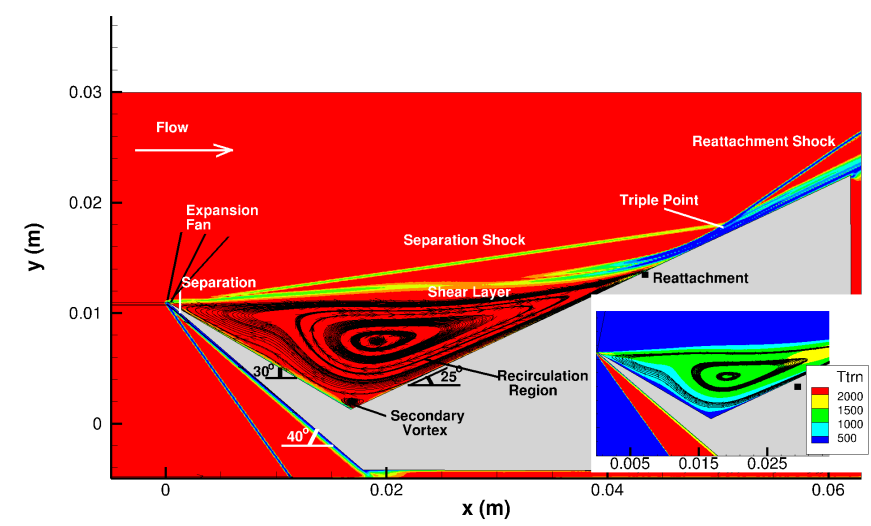

(c) Computed Schlieren image of "tick" model at $1 \mathrm{~ms}$ with the inset of translational temperature contours $\mathrm{K}$.

FIGURE 1. Shock structure of the different geometries.

\section{RESULTS AND DISCUSSION}

\section{Velocity and Temperature Slip}

The characterization of the effects of the slip velocity and temperature jump in the separation region provides an opportunity to test the fidelity of the simulations for modeling the key flow physics such as gas-surface interactions as well as establish numerical convergence in near-continuum flow conditions. Reference [8] in fact used the slip velocity as a convergence criterion for the double cone simulations due to its sensitivity to the DSMC numerical parameters. The slip velocity $\left(V_{s}\right)$ is related to the incident $\left(V_{i}\right)$ and reflected $\left(V_{r}\right)$ velocities of the molecules that strike the surface element as:

$$
V_{S}=\left[\frac{\sum_{j=1}^{N_{h i t}}\left(\frac{V_{\tau j i}}{\left|V_{n j i}\right|}+\frac{V_{\tau j r}}{\left|V_{n j r}\right|}\right)}{\sum_{j=1}^{N_{h i t}}\left(\frac{1}{\left|V_{n j i}\right|}+\frac{1}{\left|V_{n j r}\right|}\right)}\right]
$$


TABLE 2. DSMC Numerical Parameters for the simulations

\begin{tabular}{lcccc}
\hline \multicolumn{1}{c}{ Numerical Parameters: } & Nitrogen & Air & Argon & Tick (Air) \\
\hline Total number of time-steps & 800,000 & 300,000 & 300,000 & 600,000 \\
Time step, s & $1.0 \times 10^{-9}$ & $1.0 \times 10^{-9}$ & $1.0 \times 10^{-9}$ & $2.0 \times 10^{-9}$ \\
Number of simulated particles & $1.155 \times 10^{9}$ & $5.7 \times 10^{9}$ & $0.944 \times 10^{9}$ & $1.1 \times 10^{9}$ \\
Total number of collision cells & $3.05 \times 10^{8}$ & $3.44 \times 10^{8}$ & $3.45 \times 10^{8}$ & $47 \times 10^{6}$ \\
Number of processors (CPUs) & 960 & 960 & 640 & 512 \\
Total computation hours (CPU hours) & 46,080 & 12,180 & 27,870 & 141,000 \\
\hline
\end{tabular}

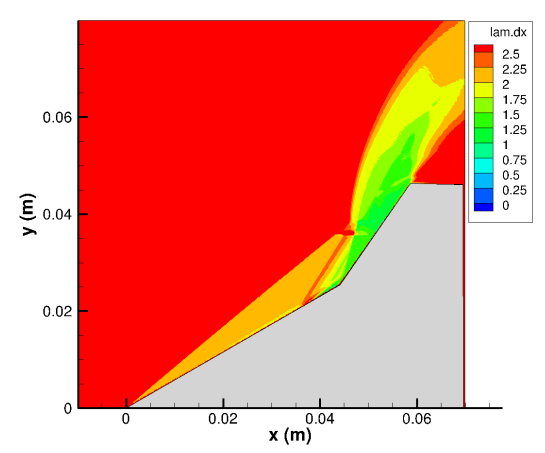

(a) The value of the ratio of the mean free path to the collision cell size

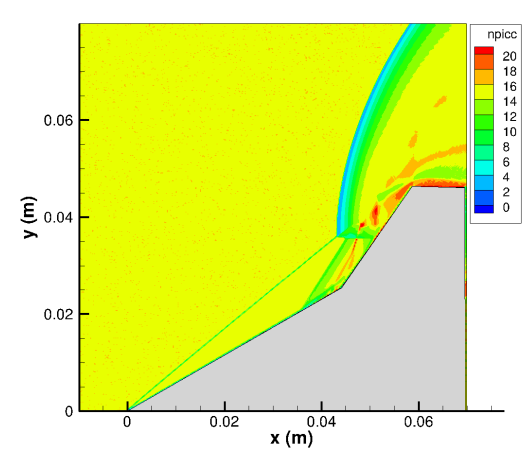

(b) The number of particles in collision cell

FIGURE 2. Spatial distribution of the numerical parameters at $100 \mu$ s for the air case.

where $\tau$ and $n$ stand for the velocity component tangential and normal to the surface, respectively. Similarly, the surface translational temperatures in the normal $\left(T_{t r n, n}\right)$, tangential $\left(T_{t r n, \tau}\right)$ and lateral $\left(T_{t r n, L}\right)$ directions can be calculated by:

$$
T_{t r n, d}=\frac{m}{k}\left[\frac{\sum_{j=1}^{N_{h i t}}\left(\frac{V_{d j i}^{2}}{\left|V_{n j i}\right|}+\frac{V_{d j r}^{2}}{\left|V_{n j r}\right|}\right)}{\sum_{j=1}^{N_{h i t}}\left(\frac{1}{\left|V_{n j i}\right|}+\frac{1}{\left|V_{n j r}\right|}\right)}-V_{d}^{2}\right]
$$

where $d$ stands for $n, \tau, L$ directions. The translational temperature jump is calculated by subtracting the wall temperature from the temperature value obtained by averaging over the surface temperatures in the aforementioned directions. The surface rotational and vibrational temperatures can be calculated in a similar fashion. That is,

$$
T_{f}=\frac{2}{\zeta_{f} k}\left[\frac{\sum_{j=1}^{N_{h i t}}\left(\frac{e_{f j i}}{\left|V_{n j i}\right|}+\frac{e_{f j r}}{\left|V_{n j r}\right|}\right)}{\sum_{j=1}^{N_{h i t}}\left(\frac{1}{\left|V_{n j i}\right|}+\frac{1}{\left|V_{n j r}\right|}\right)}\right]
$$

where $e$ denotes internal energy of particles striking the surface element and $f$ represents either rotational or vibrational mode.

Figure 3(a) shows a comparison of the slip velocities for the three sets of DSMC numerical parameter cases (i.e. the "baseline case" is presented in Table 2, the "baselineplus case" corresponds to two times more particles in comparison to the baseline case and the case labeled as "baseline case with $\Delta t / 2$ " is obtained by just halving the time step of the baseline case). The prediction of the slip velocity for the cases are essentially the same, indicating that the 2-D simulations are converged with cell, particle and time step. The length of the separation region can be determined by taking the difference of the two locations (i.e. S and R) where the slip velocity is zero, as shown in Fig. 3 (a). The 


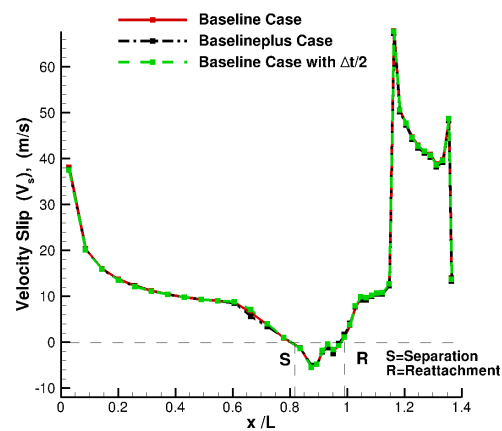

(a) Velocity slip for the double wedge (Nitrogen)(b) Temperature jump for the double wedge (Niat $100 \mu \mathrm{s}$

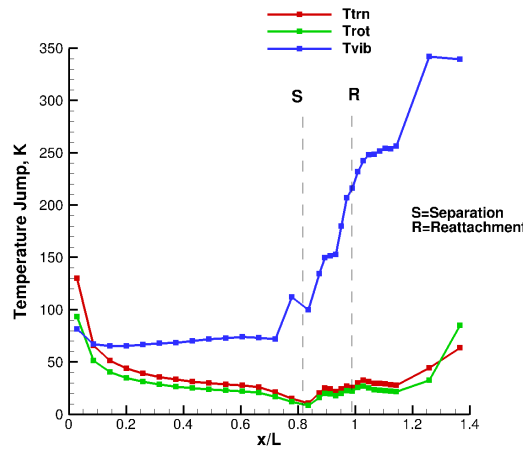

trogen) at $100 \mu$ s

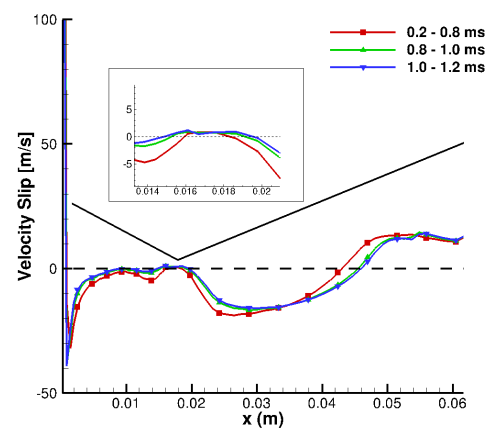

(c) Velocity slip for "tick" model

FIGURE 3. Variation of velocity slip with the number of particles and time, and temperature jump values.

slip velocity values near the tip of the wedge are found to be larger due to significant rarefaction effects at the sharp leading edge and decrease in the downstream direction, especially in the separation region. Similarly, temperature jump values slightly decrease along the first wedge surface and increase at the second wedge surface. Interestingly, the degree of the nonequilibrium in vibrational mode is found to be larger in comparison to rotational one, as shown in Fig. 3(b). However, the vibrational temperature is too small to have an effect on the surface heating with the different surface accommodation coefficients.

Similarly, although the velocity values are small because of the large separation region, a substantial amount of the slip is observed in the "tick" model configuration due to rarefaction effects resulting from sudden expansion of the flow at the leading edge, as can be seen in Fig. 3 (c). It was observed that the size of the primary vortex as well as the secondary vortex (Moffatt eddies) increases in time until the flow reaches steady state. It should be noted that the boundary layer at around $x=0.015 \mathrm{~m}$ is found to be somewhat thicker (refer to the inset of Fig. 1(c)); however, in contrast to the recent CFD calculations [16], a secondary vortex does not shift at this location forming a large secondary vortex in this DSMC calculation. The differences may be attributed to the modeling of the slip velocity. It is important to note that the run time of the experiment is about $1 \mathrm{~ms}$ which is comparable with that of the simulation.

\section{Results of Reacting Air Flow}

For the pure nitrogen case, molecular nitrogen dissociation was too small to affect the surface heat flux values for the $M=7$ double wedge case. However, due to the lower dissociation energy of molecular oxygen it is expected that this chemical reaction will affect the flowfield properties and shock structure significantly. Therefore, the $\mathrm{O}_{2}$ dissociations through collisions with nitrogen and oxygen molecules, "Case 2", and with inclusion of the exchange reaction $\left(\mathrm{N}_{2}\right.$ $+\mathrm{O} \longrightarrow \mathrm{NO}+\mathrm{N}$ ) to the dissocoation reactions denoted as "Case 1" are modeled in the current study.

Similar to the non-reacting nitrogen case, the Edney type V strong shock interactions can also be seen in this case, as shown in Fig. 1(b). For the air case, the separation shock formed on the front wedge is found to be closer to the hinge location than the nitrogen case which results in a smaller shock induced separation region. More specifically, the impingement point is at $x=0.040 \mathrm{~m}$ for this case whereas this point is at $0.035 \mathrm{~m}$ in nitrogen case as seen in Fig. 1(a) at $100 \mu \mathrm{s}$. Also the distance between the bow shock and shear layer is smaller in the air case due to chemical reactions, which is consistent with the experiment. It should be noted that no recombination reactions are taken into consideration in this study.

The complex shock structure results in thermal non-equilibrium consistent with the flow Mach number. Figures 4(a) and (b) show the spatial distribution of the translational temperature at $300 \mu$ s for the air and nitrogen case, respectively. As expected, the translational temperature values are lower than the nitrogen case because the endothermic chemical reactions remove energy from the flow. The profiles of the temperature are somewhat similar to the nitrogen case throughout the whole domain; however, the high-temperature region downstream of the bow shock is thinner for the air case. In fact, after $200 \mu$ s, the temperature profile and values do not change significantly, the growth of the separation bubble size stops, in contrast to the 2-D nitrogen case in which the temperature values keep changing 


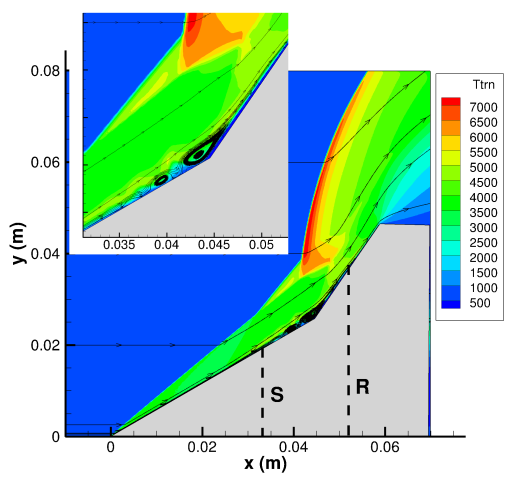

(a) Air case

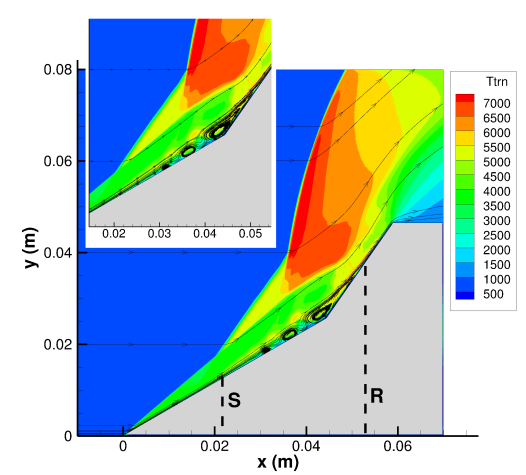

(b) Nitrogen case

FIGURE 4. Translational temperature $[\mathrm{K}]$ at $300 \mu \mathrm{s}$, 2-D model.

until $500 \mu$ s. Additionally, for the air case, the movement of the bow shock towards to the tip of the wedge stops after $200 \mu$ s. Based on the experimental shock transient profiles (not shown), the movement of the triple point towards to the leading edge in the air case is less than for the nitrogen case. These simulation results show similar trends with the experiment. Overall, in contrast to the nitrogen 2-D case, a fair agreement is achieved with the 2-D wedge model in terms of transient shock profiles. This is due the fact that the endothermic chemical reactions reduce the size of the separation region as shown in the insets of Fig. 4 which in turn leads to the reduction of three-dimensional effects.

Figure 5 illustrates the time dependence of the heat flux values. As shown in Fig. 5 (a) the measured and computed values for the air case are in close agreement at the front part of the wedge; however, discrepancies are observed at the aft part of the wedge, especially for the peak values in contrast to the nitrogen case. As can be seen in Fig. 5(b), the heat flux values are found to be in a good agreement at earlier time for the nitrogen case. The underprediction of the maxima for the air case may be explained by the lack of the modeling of recombination of the species at the surface. Previous studies $[3,4,17]$ showed that the exothermic recombination reactions enhance the heat flux values. Overall, the trend of the heat flux is found to be in fair agreement in the air 2-D dimensional case up to $200 \mu \mathrm{s}$. At later times; however, the differences in the profiles and values increase both for the air and nitrogen cases, which may be due to an increase of the separation size. Although the size of the separation region is smaller than that of the nitrogen case, the three-dimensional effects may still influence the highly sensitive heat flux values. Additional simulations were performed to investigate the effect of the exchange reaction creating the trace species (i.e. NO and $\mathrm{N}$ ) on the heat transfer rates. As can be seen in Fig. 5(a), a small difference at the peak values is observed at the aft part of the wedge in the computed heat flux values so that these values are relatively insensitive to the existence of the atomic nitrogen and NO. Lastly, the growth of the size of the separation was computed in the "tick" configuration, as shown in Fig. 5(c). Specifically, the maxima of heat flux values moves in the downstream direction due to the fact that the size of the primary vortex becomes larger in time.

\section{Effects of Gas Species}

To investigate the effects of the internal mode thermal relaxation on the shock structure, an argon gas is simulated using the same flow conditions given in Table 1 and the numerical parameters are presented in Table 2 for the double wedge geometry. The translational temperature after the bow shock of about $16,000 \mathrm{~K}$ is much larger compared to the nitrogen and air cases because no energy is absorbed in excitation of the internal modes or due to chemical reactions. Due to the strong detached shock, most of the region is subsonic. As a result, the shear layer and the transmitted and separation shocks are not as distinctive as in the nitrogen and air cases shown in Figs. 1(a) and 1(b), respectively. Moreover, the size of the separation region is also larger in comparison to the nitrogen and air cases. More specifically, as shown in Fig. 6(a), the size of the separation for argon, nitrogen and air at 100 $\mu$ s are about 0.018, 0.0101 and 0.0066 $\mathrm{m}$, respectively. The size of the separation has an impact on the position of the triple point, as shown in Fig 6(b). To understand the effect of the internal modes and chemical reactions on the surface properties more closely, the heat transfer rates at $100 \mu \mathrm{s}$ are shown in Fig. 6(c). Due to the fast movement of the triple point towards to the leading edge for the argon case, the heat flux values at the front face of the wedge would be higher in comparison to the 


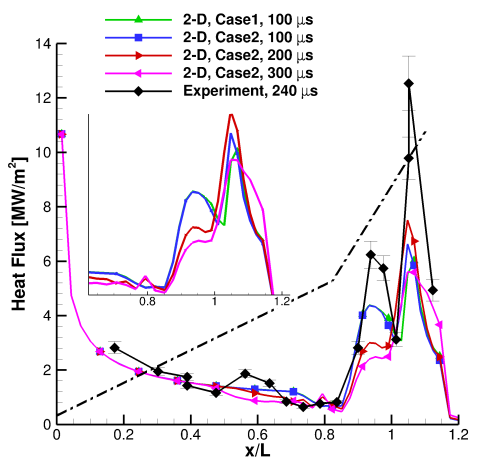

(a) Double wedge, Air

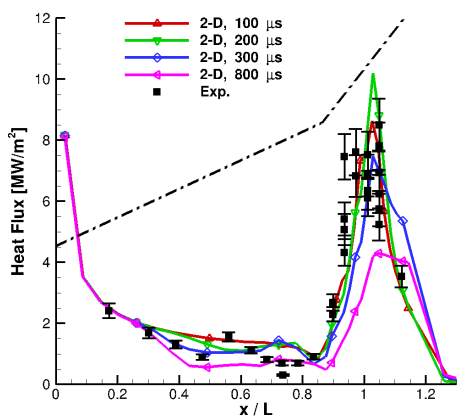

(b) Double wedge, Nitrogen

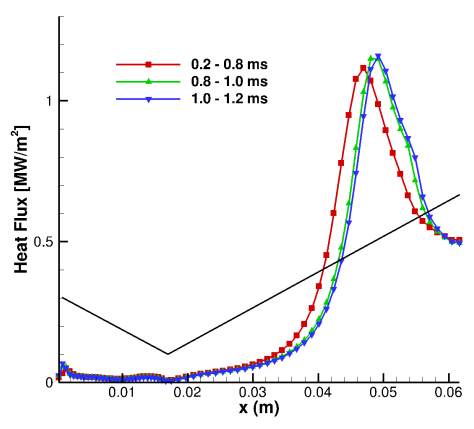

(c) "Tick" model, Air

FIGURE 5. Time evolution of surface heat flux values over 2-D geometries.

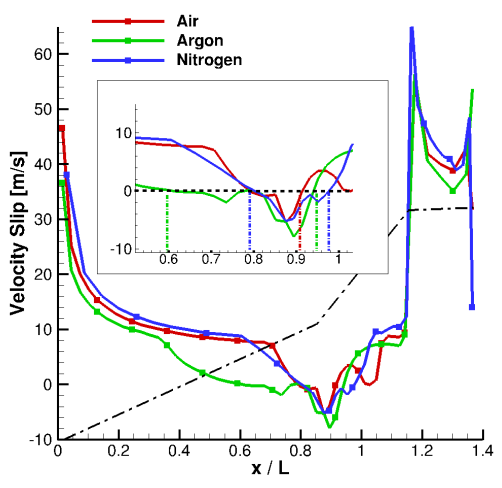

(a) Velocity slip

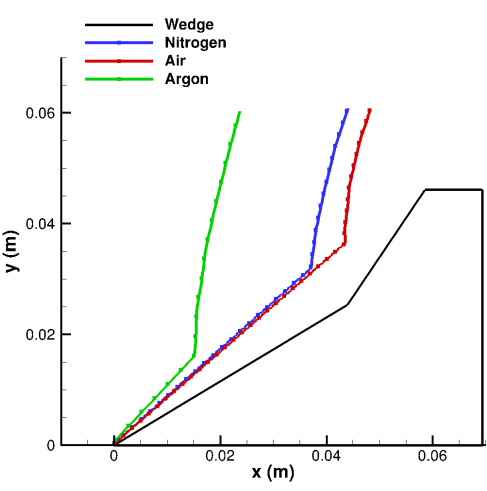

(b) Transient shock profile

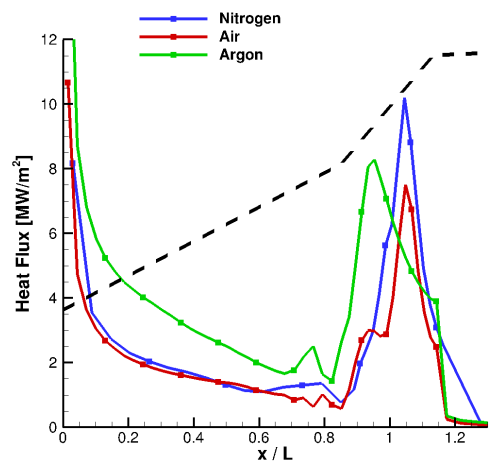

(c) Heat transfer rates

FIGURE 6. Variation of the surface parameters and shock profile for different species at $100 \mu \mathrm{s}$.

nitrogen and air cases. Comparing the heat flux of the nitrogen and air cases reveals that the surface heating values were significantly reduced by the endothermic reactions, consistent with the observation of Furumoto et al. [18]. For all three flow cases, the temperature values decrease at the starting point of the shear layer due to substantial energy dissipation by viscous flows. Additionally, from these results, it is expected that the effects of three-dimensionality on flowfield properties may be even stronger in the argon case as compared to nitrogen.

\section{CONCLUSIONS}

The hypersonic flow was simulated by the time accurate DSMC method using the two-dimensional model of the double wedge and "tick" configuration for different species. The numerical nitrogen and air results were compared with experiments conducted in the HET facility for a high-enthalpy nitrogen and air flow corresponding to a free stream Mach number of approximately seven. Although Knudsen number is low for both geometries, a substantial amount of the slip and temperature jump is observed. A chemically reacting air flow over the double wedge was simulated and it was observed that the separation length and the movement of the triple point toward to the leading edge is less compared with the nitrogen case. The measured and computed heat flux values were found to be in good agreement at the front part of the wedge. The movement of the triple point towards to the leading edge for the argon case is found to be fastest in comparison to the nitrogen and air cases. For both models, the surface is assumed to be non-catalytic with fully diffusive and a constant surface temperature of $300 \mathrm{~K}$.

To improve the modeling, surface chemical reactions such as nitridation, oxidation and catalytic recombination 
will be considered in future work. Based on the numerical results shown for the "tick" configuration and the double wedge geometry, flow separation significantly alters the stability characteristics of hypersonic flows. The temporal stability analysis will be conducted based on numerical results obtained by the DSMC method in order to determine the time constant for steadiness and the calculated results will be compared with the experiment by the aid of the visualization techniques (i.e. PLIF and RESI) for the "tick" model. The influence of the various physical and numerical parameters on the stability will also be identified. Furthermore, the measured surface parameters for the "tick" geometry will be compared with DSMC and NS solutions as well.

\section{ACKNOWLEDGMENTS}

The research is being performed at the University of Illinois Urbana-Champaign is supported by the Air Force Office of Scientific Research through AFOSR Grant No. FA9550-11-1-0129 with a subcontract award number 2010-0617101 to UIUC. This research is also a part of the Blue Waters sustained-petascale computing project, which is supported by the National Science Foundation (awards OCI-0725070 and ACI-1238993) and the state of Illinois. Blue Waters is a joint effort of the University of Illinois at Urbana-Champaign and its National Center for Supercomputing Applications.

\section{REFERENCES}

[1] J. P. Davis and B. Sturtevant, Physics of Fluids 12, 2661-2686 (2000).

[2] J. C. Robinet, J. Fluid Mech. 579, 85-112 (2007).

[3] C. Ballaro and J. Anderson Jr, "Shock strength effects on separated flows in non-equilibrium chemically reacting air shock wave/boundary layer interaction," AIAA paper 91-0250 (1991).

[4] A. Grumet, J. Anderson, and M. Lewis, "A numerical study of the shock wave/ boundary layer interaction for nonequilibrium chemically reacting air: the effects of catalytic walls," AIAA paper 91-0245 (1991).

[5] J. N. Moss, "Dsmc computations for regions of shock/shock and shock/boundary layer interaction," AIAA paper 2001-1027 (2001).

[6] G. N. Markelov, M. S. Ivanov, S. F. Gimelshein, and D. A. Levin, Rarefied Gas Dynamics 23rd International Symposium 431-440 (2003).

[7] M. S. Holden and T. P. Wadhams, "Code validation study of laminar shock/boundary layer and shock/shock interactions in hypersonic flow. part a: Experimental measurements,” AIAA paper 2001-1031 (2001).

[8] J. N. Moss and G. A. Bird, AIAA Journal 43, 2565-2573 (2005).

[9] J. Moss, S. O'Byrne, N. Deepak, and S. Gai, "Simulations of hypersonic, high-enthalpy separated flow over a'tick'configuration," in 28th International Symposium on Rarefied Gas Dynamics 2012, Vol. 1501 (AIP Publishing, 2012), pp. 1453-1460.

[10] A. Swantek and J. Austin, AIAA Journal 53, 311-320 (2014).

[11] D. R. Chapman, D. M. Kuehn, and H. K. Larson, "Investigation of separated flows in supersonic and subsonic streams with emphasis on the effect of transition," NACA-TR-1356 (1958).

[12] J. Moss, S. O’Byrne, and S. Gai, "Hypersonic separated flows about" tick" configurations with sensitivity to model design," in 29th International Symposium on Rarefied Gas Dynamics 2014 (AIP Publishing, 2014).

[13] M. S. Ivanov, G. N. Markelov, and S. G. Gimelshein, "Statistical simulation of reactive rarefied flows numerical approach and applications," AIAA paper 1998-2669 (1998).

[14] M. S. Ivanov and S. V. Rogasinsky, Soviet Journal of Numerical Analysis and Mathematical Modeling 3, 453-465 (1988).

[15] G. A. Bird, Molecular Gas Dynamics and the Direct Simulation of Gas Flows (Clarendon, Oxford, England, U.K., 1994).

[16] A. Khraibut, S. Gai, and A. Neely, "A numerical study of hypersonic laminar leading edge separation with emphasis on wall temperature and real gas effects," in 51st International Conference on Applied Aerodynamics (2016).

[17] M. MacLean, A. Dufrene, and M. Holden, "Spherical capsule heating in high enthalpy carbon dioxide in LENS-XX expansion tunnel," AIAA paper 2013-0906 (2013).

[18] G. H. Furumoto, X. Zhong, and J. C. Skiba, Physics of Fluids 9, 191-210 (1997). 